\title{
A Hybrid of Genetic Algorithm and Support Vector Machine for Feature Reduction and Detection of Vocal Fold Pathology
}

\author{
Vahid Majidnezhad \\ The United Institute of Informatics Problems, National Academy of Science of Belarus \\ Minsk, Belarus \\ vahidmn@yahoo.com \\ Igor Kheidorov \\ The United Institute of Informatics Problems, National Academy of Science of Belarus \\ Minsk, Belarus \\ ikheidorov@sakrament.com
}

\begin{abstract}
Acoustic analysis is a proper method in vocal fold pathology diagnosis so that it can complement and in some cases replace the other invasive, based on direct vocal fold observation, methods. There are different approaches and algorithms for vocal fold pathology diagnosis. These algorithms usually have three stages which are Feature Extraction, Feature Reduction and Classification. While the third stage implies a choice of a variety of machine learning methods (Support Vector Machines, Artificial Neural Networks, etc), the first and second stages play a critical role in performance and accuracy of the classification system. In this paper we present initial study of feature extraction and feature reduction in the task of vocal fold pathology diagnosis. A new type of feature vector, based on wavelet packet decomposition and Mel-Frequency-Cepstral-Coefficients (MFCCs), is proposed. Also a new GA-based method for feature reduction stage is proposed and compared with conventional methods such as Principal Component Analysis (PCA). Support vector machine is used as a classifier for evaluating the performance of the proposed method. The results show the priority of the proposed method in comparison with the current methods.
\end{abstract}

Index Terms - Vocal Fold Pathology Diagnosis, Wavelet Packet Decomposition (WPD), Mel-FrequencyCepstral-Coefficient (MFCC), Principal Component Analysis (PCA), Genetic Algorithm (GA), Support Vector Machine (SVM)

\section{INTRODUCTION}

Speech signal information often plays an important role for specialists to understand the process of vocal fold pathology formation. In some cases speech signal analysis can be the only way to analy ze the state of vocal folds.

Nowadays diverse medical techniques exist for direct examination and diagnose of pathologies. Laryngoscopy, electro myography, videokimography are most frequently used by medical specialists. But these methods possess a number of disadvantages. Human vocal tract is hardly accessible for visual examination during phonation process and that makes it more problematic to identify pathology. Moreover, these diagnostic means may cause the patients feel much discomfort and distort the actual signal so that it may be lead to incorrect diagnosis as well [1-4].

Acoustic analysis as a diagnostic method has no drawbacks, peculiar to the above mentioned methods. It possesses a number of advantages. First of all, acoustic analysis is a non-invasive diagnostic technique that allows pathologists to examine many people in short time period with minimal discomfort. It also allows pathologists to reveal the pathologies on early stages of their origin. This method can be a great interest for medical institutions. In recent years a number of methods were developed for segmentation and classification of speech signals with pathology.

Different parameters for feature extraction are used. Traditionally, one deals with such parameters like pitch, jitter, a mp litude perturbation, pitch perturbation, signal to noise ratio, normalized noise energy [5] and others [6-9]. Feature extraction, using the above mentioned parameters, has shown its efficiency for a number of practical tasks [8]. These parameters are frequently used in systems for automatic vocal fold pathology diagnosis, in speaker identification systems or in multimedia database indexing systems. In the proposed method, the Mel-Frequency-Cepstral-Coefficients (MFCCs), Energy and Shannon Entropy parameters have been used for creating the features vector.

Also different approaches for feature reduction are used such as Principal Component Analys is (PCA) [1013] and Linear Discriminant Analysis (LDA) [14]. In the proposed method, the GA-based feature reduction has been used and the results of experiments show its better performance in comparison with the PCA.

Finally, the reduced features are used for speech classification into the healthy and pathological class. 
Different machine learning methods such as Support Vector Machines [10], Artificial Neural Networks [15], Hidden Markov Model [9], etc can be used as a classifier. In the proposed method, the SVM has been used for the classification purpose. In table I, some pervious methods are summarized.

The rest of the paper is organized as follows. In the section II, the MFCC and WPD as the feature extraction tools are described. Also the well-known PCA and the proposed GA methods as the feature reduction methods are described. Also, the SVM as the classification tool is described. Experimental results and analysis are summarized in the section III. The section IV concludes the paper.

TABLE I. THE SUMMARY OF SOME PERVIOUS WORKS

\begin{tabular}{|c|c|c|c|}
\hline Reference & Feature set & Feature Reduction Method & Classifier \\
\hline$[10]$ & 25 Acoustic parameters given by MDVP & PCA & Support Vector Machine \\
\hline$[11]$ & Spectral perturbation & PCA & K-Means clustering \\
\hline$[12]$ & Acoustic feature, noise & PCA & Threshold \\
\hline$[13]$ & Linear prediction coefficients & PCA & Gaussian M ixture Model \\
\hline$[14]$ & Mel-frequency-cep stral-coefficients & - & Artificial Neural Network \\
\hline$[15]$ & Spectral & & LA \\
\hline
\end{tabular}

\section{METHODOLOGY}

The Block diagram of the proposed method is illustrated in the figure 1 . In the first stage, by the use of MFCC and Wavelet Packet Decomposition, feature vector containing 139 features is made. In the second stage, by the use of the proposed GA-based method, the dimension of feature vector is reduced. In the last stage, by the use of Support Vector Machines (SVM), the speech signal classified into two classes: pathological or healthy.

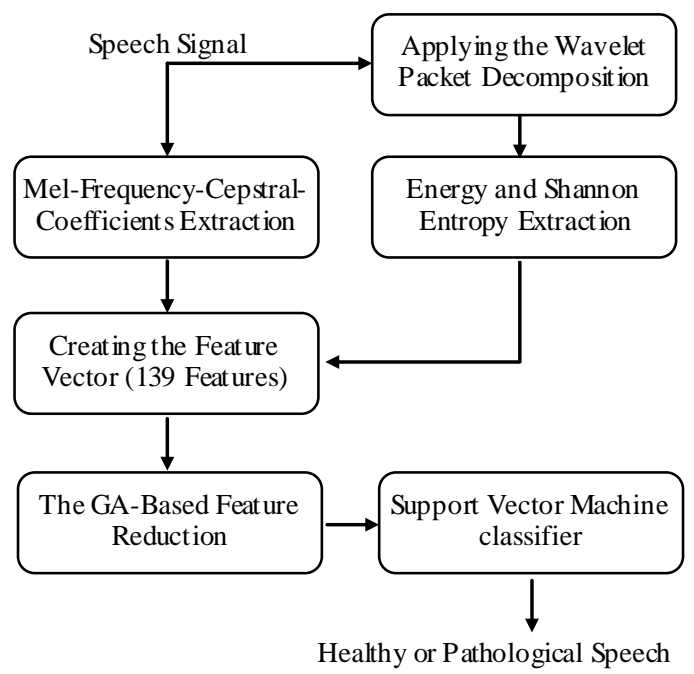

Figure 1. The scheme of the proposed method

\section{A. Feature Extraction}

As it is shown in the figure 1, first, by the use of cepstral representation of input signal, 13 MelFrequency-Cepstral-Coefficients (MFCC) are extracted. Then the wavelet packet decomposition in 5 levels is applied on the input signal to make the wavelet packet tree. Then, from the nodes of resulting wavelet packet tree, 63 energy features along with 63 Shannon entropy features are extracted. Finally, by the combination of these features, the initial feature vector with the length of 139 features is created.
Mel-Frequency-Cepstral-Coefficients

(MFCCs): MFCCs are widely used features to characterize a voice signal and can be estimated by using a parametric approach derived from linear prediction coefficients (LPC), or by the non-parametric discrete fast Fourier transform (FFT), which typically encodes more information than the LPC method. The signal is windowed with a Hamming window in the time domain and converted into the frequency domain by FFT, which gives the magnitude of the FFT. Then the FFT data is converted into filter bank outputs and the cosine transform is found to reduce dimensionality. The filter bank is constructed using 13 linearly-spaced filters (133.33Hz between center frequencies,) followed by 27 log-spaced filters (separated by a factor of 1.0711703 in frequency.) Each filter is constructed by combining the amplitude of FFT bin.

The Matlab code to calculate the MFCC features was adapted from the Auditory Toolbox (Malcolm Slaney). The MFCCs are used as features in [14] to classify the speech into pathology and healthy class. The MFCC information has been reduced by averaging the sample's value of each coefficient.

Wavelet Packet Decomposition (WPD): Recently, wavelet packets (WPs) have been widely used by many researchers to analyze voice and speech signals. There are many out-standing properties of wavelet packets which encourage researchers to employ them in widespread fields.

The most important, multi resolution property of WPs is helpful in voice signal synthesis [16-17]. The hierarchical WP transform uses a family of wavelet functions and their associated scaling functions to decompose the original signal into subsequent sub-bands. The decomposition process is recursively applied to both the low and high frequency sub-bands to generate the next level of the hierarchy. WPs can be described by the following collection of basic functions:

$$
W_{2 n}\left(2^{p-1} x-1\right)=\sqrt{2^{1-p}} \sum_{m} h(m-2 l) \sqrt{2^{p}} W_{n}\left(2^{p} x-m\right)
$$




$$
W_{2 n+1}\left(2^{p-1} x-1\right)=\sqrt{2^{1-p}} \sum_{m} g(m-2 l) \sqrt{2^{p}} W_{n}\left(2^{p} x-m\right)
$$

where $p$ is scale index, $l$ the translation index, $h$ the lowpass filter and g the high-pass filter with

$$
g(k)=(-1)^{k} h(1-k)
$$

the WP coefficients at different scales and positions of a discrete signal can be computed as follows:

$$
\begin{aligned}
& C_{n, k}^{p}=\sqrt{2^{p}} \sum_{m=-\infty}^{\infty} f(m) W_{n}\left(2^{p} m-k\right) \\
& C_{2 n, l}^{p-1}=\sum_{m} h(m-2 l) C_{n, m}^{p} \\
& C_{2 n+1, l}^{p-1}=\sum_{m} g(m-2 l) C_{n, m}^{p}
\end{aligned}
$$

for a group of wavelet packet coefficients, energy feature in its corresponding sub-band is computed as

$$
\text { Energy }_{n}=\frac{1}{N^{2}} \sum_{k=1}^{n}\left|C_{n, k}^{p}\right|^{2}
$$

The entropy evaluates the rate of information which is produced by the pathogens factors as a measure of abnormality in pathological speech. Also, the measure of Shannon entropy can be computed using the extracted wavelet-packet coefficients, through the following formula

$$
\text { Entropy }_{n}=-\sum_{k=1}^{n}\left|C_{n, k}^{p}\right|^{2} \log \left|C_{n, k}^{p}\right|^{2}
$$

In this study, mother wavelet function of the tenth order Daubechies has been chosen and the signals have been decomposed to 5 levels. The mother wavelet used in this study is reported to be effective in voice signal analysis [18-19] and is being widely used in many pathological voice analyses [17]. Due to the noise-like effect of irregularities in the vibration pattern of damaged vocal folds, the distribution manner of such variations within the whole frequency range of pathological speech signals is not clearly known. Therefore, it seems reasonable to use WP rather than DWT or CWT to have more detail sub-bands.

\section{B. Feature Reduction}

Using every feature for classification process is not good idea and it may be causes to the increasing the rate of misclassification. Therefore, it is better to choose the proper features from the whole features. This process is called as "Feature Reduction".
In other words, the goal is to reduce the dimension of the data by finding a small set of important features which can give good classification performance. One way for feature reduction is Principal Component Analysis (PCA) which is used frequently in pervious works such as [10-13]. In this section also a novel approach, the GA-based Feature Reduction, is proposed for the feature reduction stage.

Principal Component Analysis (PCA): This method searches a mapping to find the best representation for distribution of data. Therefore, it uses a signalrepresentation criterion to perform dimension reduction while preserving much of the randomness or variance in the high-dimensional space as possible [20].

The first principal component accounts for as much of the variability in the data as possible, and each succeeding component accounts for as much of the remaining variability as possible. PCA involves the calculation of the eigenvalues decomposition of a data covariance matrix or singular value decomposition of a data matrix, usually after mean centering the data for each attribute.

PCA is mathematically defined as an orthogonal linear transformation that transforms the data to a new coordinate system such that the greatest variance by any projection of the data comes to lie on the first coordinate, called the first principal component, the second greatest variance on the second coordinate, and so on.

The principal component $W_{1}$ of a dataset $X$ can be defined as:

$W_{1}=\arg _{\|w\|=1} \max \operatorname{var}\left\{W^{T} X\right\}=\arg _{\|w\|=1} \max E\left\{\left(W^{T} X\right)^{2}\right\}(9)$

With the first $K-1$ components, the $K^{\text {th }}$ component can be found by subtracting the first $K-1$ principal components from $X$ :

$$
\widehat{X}_{k-1}=X-\sum_{i=1}^{k-1} W_{i} W_{i}^{T} X
$$

and by substituting this as the new data set to find a principal component in,

$$
W_{k}=\arg _{\|w\|=1} \max E\left\{\left(W^{T} \widehat{X}_{k-1}\right)^{2}\right\}
$$

The Karhunen Leove transform is therefore equivalent to finding the singular value decomposition of the data matrix, $X$.

$$
X=W \sum V^{T}
$$

and then obtaining the reduced-space data matrix by $Y$ projecting $X$ down into the reduced space defined by only the first $L$ singular vectors, $W_{L}$ :

$$
Y=W_{L}^{T} X=\sum V_{L}^{T}
$$


The matrix $W$ of singular vectors of $X$ is equivalently the matrix $W$ of eigenvectors of the matrix of observed co-variances, $C=X X^{T}$,

$$
X X^{T}=W \sum \sum W^{T}
$$

In PCA, the optimal approximation of a random vector $X \in R^{N}$ in $N$-dimensional space by a linear co mbination of $M(M<N)$ independent vectors is obtained by projecting the random vector $X$ into the eigenvectors corresponding to the largest eigenvalues of the covariance matrix of vector $X$ [20].

The main limitation of PCA is that it does not consider class separately, since it does not take into account the class label of the feature vectors.

GA-Based Feature Reduction: Genetic Algorithm (GA) is a heuristic optimization method which acts on the basis of evaluation in nature and search for the final solution among a population of potential solutions. It has three basic operations which are mutation, crossover and selection. Recently, it is used widely in optimizations problems such as [21].

Genetic Algorithms act on the basis of evaluation in nature search for the final solution among a population of potential solution. In every generation the fittest of that generation selected and after reproduction produce a new set of children. In this process the fittest individuals will survive more probably to the next generations.

At the beginning of algorithm a number of individuals (initial population) are created randomly and the fitness function is evaluated for all of them. If we do not reach to the optimal answer, the next generation is produced with selection of parents based on their fitness and the children mutates with a fixed probability then the new children fitness is calculated and new population is formed by substitution of children with parents and this process is repeated until the final condition is established.

As it is mentioned before, the main limitation of PCA is that it does not take into account the class labels and it just focus on the sample's value.

In other words, the PCA searches for the features which their sample's value have bigger variance in comparison with others and it does not collaborate with the classifier. So, for overcoming this disadvantage, by using genetic algorithm a GA-based method is proposed which considers the error rate of the classifier in its fitness function and tries to minimize it. For this purpose, a fitness function $f$ is defined which shows the error rate of the SVM classifier for the train set.

$$
f=\sum_{i=1}^{n}\left|a_{i}-r_{i}\right|
$$

The $a_{i}$ is the result of classifier and the $r_{i}$ is the real class for $i^{\text {th }}$ speech signal. The $\mathrm{n}$ is the number of speech files in the "train" dataset. The aim of the GA-based method is to find the subset of features so that they minimize the $f$.
In the proposed method, the length of each chromosome is 139 (the length of initial feature vector). Each gene in the chromosomes is related to one feature and has 2 values (zero and one). These values show that whether the respective feature participates in the classification's process or not.

\section{Support Vector Machines}

For classification, a statistical learning algorithm called support vector machine (SVM) is used. SVMs which were proposed by Vapnik [22], have become an acknowledged classification method in the task of musical genre recognition. Their usage in this task was already justified by works of Li et al. [23].

Given a set of training vectors belonging to two separate classes, $\left(x_{1}, y_{1}\right), \quad \ldots . \ldots . .,\left(x_{1}, y_{l}\right)$, where $x_{i} \in R^{N}$ and $y_{i} \in\{-1,1\}$, one wants to find a hyperplane $w x+b=0$ to separate the data. In fact, there are many possible hyper-planes, but there is only one that maximizes the marg in (the distance between the hyperplane and the nearest data point of each class). The solution to the optimization problem of SVM is given by the saddle point of the Lagrange functional

$$
L(w, b, \alpha)=\frac{1}{2}\|w\|^{2}-\sum_{i=1}^{l} \alpha_{i}\left\{y_{i}\left[\left(w . x_{i}\right)+b\right]-1\right\}
$$

where $\alpha_{i}$ are the Lagrange multipliers. Classical Lagrangian duality enables the primal problem (16) to be transformed to its dual problem, which is easier to solve. The solution is given by

$$
\begin{aligned}
& \bar{w}=\sum_{i=1}^{l} \bar{\alpha}_{i} y_{i} x_{i} \\
& \bar{b}=-\frac{1}{2} \bar{w} \cdot\left[x_{r}+x_{s}\right]
\end{aligned}
$$

where $x_{r}$ and $x_{s}$ are any two support vectors with, $\bar{\alpha}_{i}, \bar{\alpha}_{s}>0, y_{r}=1, y_{s}=-1$.

To solve the non-separable problem slack variables $\xi_{i}>0$ and a penalty function, $f\left(\xi_{i}\right)=\sum_{i} \xi_{i}$, where the $\xi_{i}$ are measures of the misclassification error. The solution is identical to the separable case except for a modification of the Lagrange multipliers as $0 \leq \alpha_{i} \leq C$, $i=1, . . l$. The choice of $C$ is not strict in practice.

In the proposed method, the linear function is used as the kernel function of the SVM. Also the SVM can realize nonlinear discrimination by kernel mapping [22], when the samples in the input space cannot be separated by any linear hyper-plane, but can be linearly separated in the nonlinear mapped feature space.

There are four typical kernel functions for the nonlinear mapping [22]: polynomial function, Gaussian radial basis function, multilayer perception and Exponential radial basis function. 


\section{EXPERIMENTS AND RESULTS}

In this section, three experiments have been designed. These experiments are simulated in the Matlab 7.11.0. The whole scheme of the proposed method is illustrated in the figure 1 . We have adopted 10 folds crossvalidation scheme to assess the generalization capabilities of the system in our experiments.

\section{A. Dataset Description}

The dataset was created by specialists from the Belarusian Republican Center of Speech, Voice and Hearing Pathologies. We have selected 75 pathological speeches and 55 healthy speeches randomly which are related to sustained vowel "a”. All the records are wave files in the PCM format.

\section{B. Results}

In the first experiment, we apply the t-test on each feature and compare $p$-value for each feature as a measure of how effective it is at separating groups. The result is shown in the figure 2 . There are about $40 \%$ of features having p-values close to zero and $60 \%$ of features having $p$-values smaller than 0.05 , means that there are about 83 features among the original 139 features which have strong discrimination power.

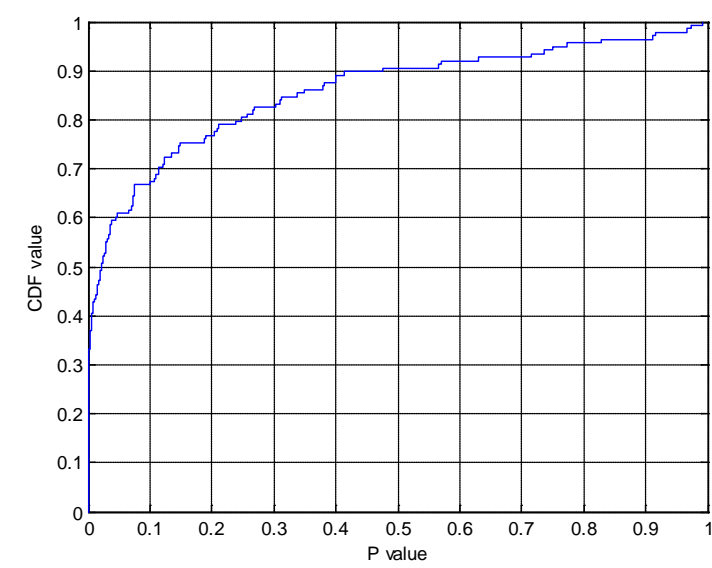

Figure 2. The P-value for the features

One can sort these features according to their p-values (or the absolute values of the t-statistic) and select some features from the sorted list. However, it is usually difficult to decide how many features are needed unless one has some domain knowledge or the maximum number of features that can be considered has been dictated in advance based on outside constraints.

One quick way to decide the number of needed features is to plot the MCE (misclassification error, i.e., the number of misclassified observations divided by the number of observations) on the test set as a function of the number of features.

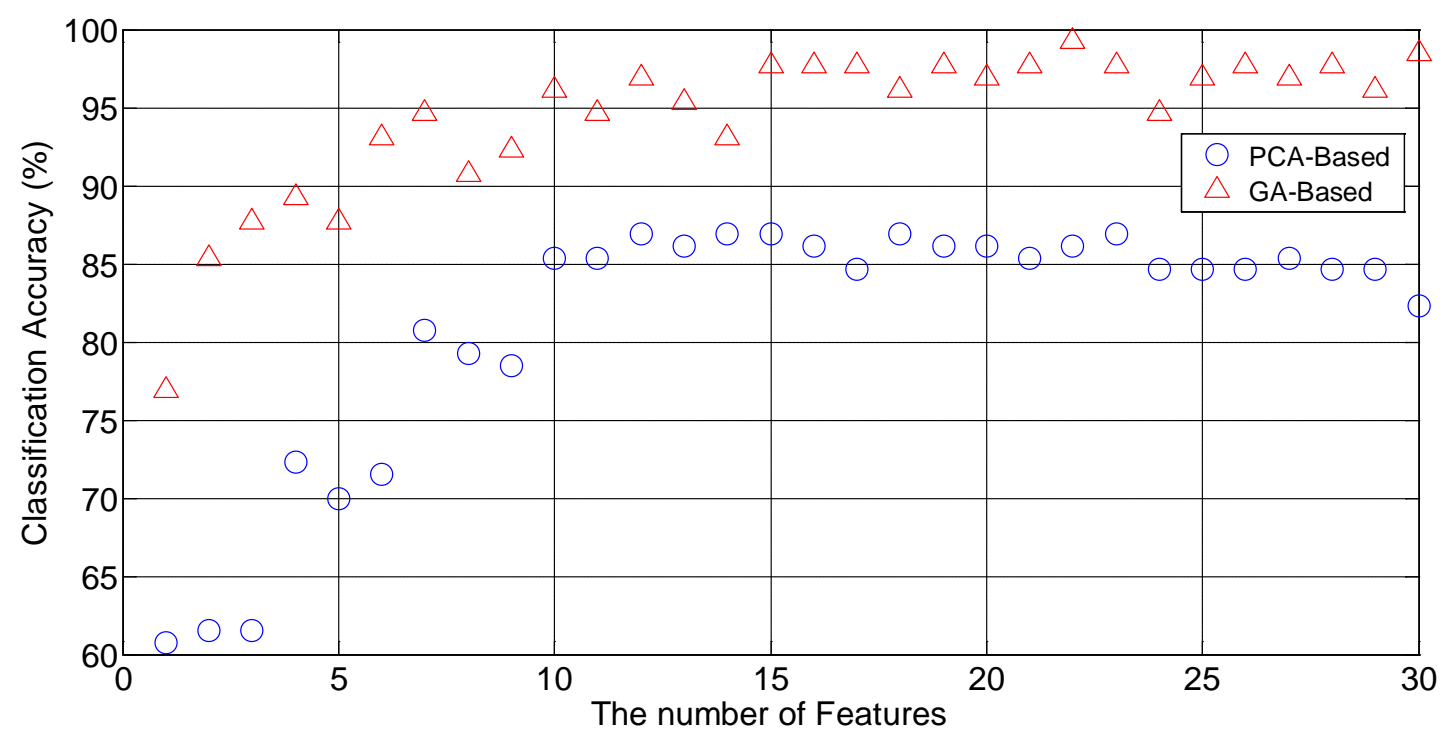

Figure 3. The obtained MCE by the means of the PCA-Based and the GA-Based Methods

In the second experiment, the PCA approach is applied for the feature reduction. Since the total number of our observations is 130 , so it is better to use the lower number of features for our classification's purpose. Therefore, the MCE has computed for various numbers of features between 1 and 30. The result is shown in the figure 3 with circular marks.

In the third experiments, the proposed GA-based method is applied for the feature reduction. The MCE has computed for various numbers of features between 1 and
30. The result is shown in the figure 3 with triangular marks.

As it is obvious in the figure 3, from the MCE point of view, the performance of the proposed GA-based approach is better than the PCA-based approach. In the best cases, the PCA-based method leads to $86.92 \%$ of accuracy with 23 selected features while the GA-based method leads to $99.23 \%$ of accuracy with 22 selected features. The selected features by the means of these methods are shown in the table II. 
TABLE II. THE selected features for the construction of feature vector in the best case

\begin{tabular}{|c|c|c|}
\hline Feat ure Reduction Method & The Selected Feat ures & Accuracy (\%) \\
\hline $\begin{array}{l}\text { The Proposed GA-Based } \\
\text { (feat ure vector length=22) }\end{array}$ & $\begin{array}{l}\text { The } 1^{\text {st }} 2^{\text {nd }} \text {, } 5^{\text {th }} \text { and } 8^{\text {th }} \text { coefficients of MFCCs. } \\
\text { Energy at the } 1^{\text {st }}, 7^{\text {th }}, 21^{\text {st }}, 28^{\text {th }}, 40^{\text {th }} \text { and } 54^{\text {th }} \text { nodes of WP Tree. } \\
\text { Entropy at the } 6^{\text {th }}, 7^{\text {th }}, 13^{\text {th }}, 24^{\text {th }}, 25^{\text {th }}, 26^{\text {th }}, 30^{\text {th }}, 32^{\text {nd }}, 34^{\text {th }}, 35^{\text {th }}, \\
60^{\text {th }} \text { and } 61^{\text {st }} \text { nodes of WP Tree. }\end{array}$ & 99.23 \\
\hline $\begin{array}{l}\text { The PCA-Based } \\
\text { (feat ure vector length=23) }\end{array}$ & $\begin{array}{l}\text { The } 1^{\text {st }}, 2^{\text {nd }}, 3^{\text {td }}, 4^{\text {th }}, 5^{\text {th }}, 6^{\text {th }}, 9^{\text {th }} \text { and } 11^{\text {th }} \text { coefficients of MFCCs. } \\
\text { Energy at the } 8^{\text {th }}, 18^{\text {th }}, 19^{\text {th }}, 22^{\text {nd }}, 23^{\text {rd }} \text { and } 25^{\text {th }} \text { nodes of WP Tree. } \\
\text { Entropy at the } 1^{\text {st }}, 2^{\text {nd }}, 4^{\text {th }}, 8^{\text {th }}, 16^{\text {th }}, 17^{\text {th }}, 32^{\text {nd }}, 35^{\text {th }} \text { and } 39^{\text {th }} \text { nodes of } \\
\text { WP Tree. }\end{array}$ & 86.92 \\
\hline
\end{tabular}

\section{Discussion}

In the first experiment, it is shown that about $40 \%$ of initial features (56 features) are not strong for the classification purpose. So, feature reduction phase is necessary and important.

Feature reduction algorithms can be roughly grouped into two categories: filter methods and wrapper methods. Filter methods rely on general characteristics of the data to evaluate and to select the feature subsets without involving the chosen learning algorithm. Wrapper methods use the performance of the chosen learning algorithm to evaluate each candidate feature subset. Wrapper methods search for features better fit for the chosen learning algorithm, but they can be significantly slower than filter methods if the learning algorithm takes a long time to run. The concepts of "filters" and "wrappers" are described in [24].

In this article, the GA-based method for the feature reduction stage has been proposed which belongs to the wrapper methods. On the other side, the Principal Component Analysis (PCA) as the one of famous filter methods has been used which is used frequently in pervious works such as [10-13].

In the experiments 2 and 3, the performances of the proposed method as a wrapper method and the PCAbased method as a filter method have been compared. In the figure 3 , it is clear that the GA-based method has better performance in comparison with the PCA-based method. This better performance is due to take into consideration of the SVM classifier in the feature reduction phase. In other words, the GA-based method tries to reduce the initial feature vector with the aim of increasing the SVM classifier accuracy. But the PCAbased method just focuses on the data without any attention on the classifier accuracy.

\section{CONCLUSION}

In this article, it is shown that features based on wavelet transformation have potential for detection of vocal fold pathology. So, in the proposed scheme, MelFrequency-Cepstral-Coefficients (MFCC) along with the wavelet packet decomposition are used for the feature extraction phase.

Also a novel approach for the feature reduction phase in the vocal fold pathology diagnosis is proposed. Three experiments are designed to investigate the efficiency of the proposed method. The proposed GA-based method achieves the $99.23 \%$ of classification accuracy. The results of experiments show the priority of the GA-based method in comparis on with the conventional PCA-based method.

Although it may be possible to try to build a complete multiclass classification system with a hierarchy of support vector machines so that detection of different type of pathological speech will be possible. For this propose, it is suggested to do further researches on more sophisticated feature extraction phase.

\section{ACKNOWLEDGMENT}

This work was supported by the speech laboratory of the United Institute of Informatics Problems of NASB in Belarus. The authors wish to thank the Belarusian Republican Center of Speech, Voice and Hearing Pathologies by its support in the speech database.

\section{REFERENCES}

[1] J.B. Alonso, J.D. Leon, I. Alonso, M.A. Ferrer, "Automatic Detection of Pathologies in the Voice by HOS Based Parameters". EURASIP Journal on Applied Signal Processing, 2001(4): 275-284, 2001.

[2] L.G. Ceballos, J. Hansen, J. Kaiser, “A Non-Linear Based Speech Feature Analysis Method with Application to Vocal Fold Pathology Assessment”. IEEE Trans. Biomedical Engineering, 45(3): 300313, 2005.

[3] L.G. Ceballos, J. Hansen, J. Kaiser, “Vocal Fold Pathology Assessment Using AM Autocorrelation Analysis of the Teager Energy Operator". ICSLP1996 Proc., pp: 757-760, 1996.

[4] C. Adnene, B. Lamia, "Analysis of Pathological Voices by Speech Processing”. Signal Processing and Its Applications, 2003 Proc., 1(1): 365-367, 2003.

[5] C. Manfredi, "Adaptive Noise Energy Estimation in Pathological Speech Signals”. IEEE Trans. Bio medical Engineering, 47(11): 1538-1543, 2000.

[6] J.I.G. Llorente, P.G. Vilda, “Automatic Detection of Voice Impairments by Means of Short-Term Cepstral Parameters and Neural Network Based Detectors”. IEEE Trans. Biomedical Engineering, 51(2): 380-384, 2004.

[7] M.D.O. Rosa, J.C. Pereira, M. Grellet, “Adaptive Estimation of Residue Signal for Voice Pathology Diagnosis”. IEEE Trans. Biomedical Engineering, 47(1): 96-104, 2000. 
[8] V. Majidnezhad, I. Kheidorov, “A Novel Method for Feature Extraction in Vocal Fold Pathology Diagnosis”. Proceeding of the 3rd International Conference on Wireless Mobile Communication and Healthcare, LNICST 61, pp: 96-105, 2013.

[9] V. Majidnezhad, I. Kheidorov, “A HMM-Based Method for Vocal Fold Pathology Diagnosis”. IJCSI International Journal of Computer Science Issues, Vol. 9, Issue 6, No 2. pp: 135-138, 2012.

[10] W. Chen, C. Peng, X. Zhu, B. Wan, D. Wei, "SVMbased identification of pathological voices". Proceedings of the 29th Annual International Conference of the IEEE EMBS. pp: 3786-3789, 2007.

[11] P. Go'mez, F. Di'az, A. A'lvarez, K. Murphy, C. Lazaro, R. Martinez, V. Rodellar, "Principal component analysis of spectral perturbation parameters for voice pathology detection". Proceedings of the 18th IEEE Symposium on Computer-Based Medical Systems, pp: 41-46, 2005.

[12] D. Michaelis, M. Frohlich, H.W. Strube, "Selection and combination of acoustic features for the description of pathologic voices". Journal of the Acoustical Society of America, 103(3): 1628-1639, 1998.

[13] M. Marinaki, C. Kotropoulos, I. Pitas, N. Maglaveras, "Automatic detection of vocal fold paralysis and edema". Proceedings of Eighth International Conference on Spoken Language Processing-ICSLP. pp: 31-32, 2004.

[14] J.I.G. Llorente, P.G. Vilda, M.B. Velasco, "Dimensionality Reduction of a Pathological Voice Quality Assessment System Based on Gaussian Mixture Models and Short-Term Cepstral Parameters”. IEEE TRANSACTIONS ON BIOMEDICA L ENGINEERING, 53(10): 1943-1953, 2006.

[15] R.T. Ritchings, M.A. McGillion, C.J. Moore, "Pathological voice quality assessment using artificial neural networks”. Medical Engineering \& Physics, 24(8): 561-564, 2002.

[16] H.K. Herisa, B.S. Aghazadeh, M.N. Bahrami, "Optimal feature selection for the assessment of vocal fold disorders". Computers in Biology and Medicine, 39(10): 860-868, 2009.

[17] E. Fonseca, R.C. Guido, J.C. Pereira, P.R. Scalassarsa, C.D. Maciel, J.C. Pereira, "Wavelet time frequency analysis and least squares support vector machines for identification of voice disorders". Computers in Biology and Medicine, 37(4): 571-578, 2007.

[18] R.C. Guido, J.C. Pereira, E. Fonseca, F.L. Sanchez, L.S. Vierira, "Trying different wavelets on the search for voice disorders sorting”. Proceedings of the 37th IEEE International Southeastern Symposium on System Theory, pp: 495-499, 2005.

[19] K. Umapathy, S. Krishnan, "Feature analysis of pathological speech signals using local discriminant bases technique". Medical and Biological Engineering and Computing, 43(4): 457-464, 2005.
[20] M.K. Arjmandi, M. Pooyan, “An optimum algorithm in pathological voice quality assessment using wavelet-packet-based features, linear discriminant analysis and support vector machine”. Biomedical Signal Processing and Control, 7(1): 3-19, 2012.

[21] V. Majidnezhad, H.M. Gader, E. Efimov, "A Novel Hybrid Algorithm for Task Graph Scheduling”. International Journal of Computer Science Issues, Vol. 8, Issue 2, pp: 32-38, 2011.

[22] V.N. Vapnik, “Statistical Learning Theory”. New York, Wiley, 1998.

[23] T. Li, M. Oginara, Q. Li, “A comparative study on content based music genre classification”. Proc. Of the 26th annual int.ACM SIGIR conf. on Research and development in information retrieval, pp: 282289, 2003.

[24] R. Kohavi, G. John, "Wrappers for feature subset selection”. Artificial Intelligence, 97(1-2): 272-324, 1997.

Vahid Majidnezhad is a PhD student at the United Institute of Informatics Problems in the National Academy of Science of Belarus. His research interests are Speech Processing and Optimization Problems.

Ig or Kheidorov is an associate professor at the United Institute of Informatics Problems in the National Academy of Science of Belarus. His research interest is Speech Processing. 